\title{
Two Dimensional Advection Diffusion Equations in Unstable Case
}

\author{
Khaled Sadek Mohamed Essa*, Sawsan Ibrahim Mohamed EI Saied \\ Department of Mathematics and Theoretical Physics, Nuclear Research Center, Egyptian Atomic Energy Authority, Cairo, Egypt \\ Email address: \\ mohamedksm56@yahoo.com (K. S. M. Essa) \\ ${ }^{*}$ Corresponding author \\ To cite this article: \\ Khaled Sadek Mohamed Essa, Sawsan Ibrahim Mohamed El Saied. Two Dimensional Advection Diffusion Equations in Unstable Case. \\ Engineering Mathematics. Vol. 5, No. 1, 2021, pp. 7-12. doi: 10.11648/j.engmath.20210501.12
}

Received: June 12, 2021; Accepted: June 29, 2021; Published: July 9, 2021

\begin{abstract}
The aim of this work is to solve the diffusion equation in two dimensions to obtain normalized crosswind integrated concentrations using the Laplace Transform technique, taking into account that the wind speed is constant but the vertical diffusivity differs from the friction velocity and the Monin -Obukhov length. A comparison of the calculated values and the observed concentrations taken from the northern part of Copenhagen, Denmark and also Inshas, Cairo, Egypt for trace hexafluoride $\left(\mathrm{SF}_{6}\right)$ through unstable condition were made. It has compared the current and observed concentration one finds that the current concentration agreement well with the observed data. The results showed an agreement between the measurements and the simulations. The values for NMSE and FB are relatively close to zero, and COR, FA2 is relatively close to one.
\end{abstract}

Keywords: Laplace Transforms Technique, Wind Speed, Advection-Diffusion Equations, Eddy Diffusivity

\section{Introduction}

Models of atmospheric dispersion were used to obtain the concentration of pollutants in the atmosphere. The term dispersion is used in this context to describe the combination of propagation (due to turbulent vortex movement) and adhesion (due to wind) occurring within the air near the surface of the earth [8]. The analytical solution of the diffusion equation for air diffusion depends on different forms of non-Gaussian solutions. An analytical solution with an energy law for wind speed and eddy diffusivities with the real assumption was examined by [3]. The analytical solution of the atmospheric diffusion equation contains different variations depending on Gaussian and non-Gaussian solutions. An analytical solution to the wind speed and eddy current power law Diffusivity with realistic assumptions is derived by Demuth [12] and Essa. [13]. Most of the fundamental theories of atmospheric diffusion were proposed in the first half of the twentieth century. The term "atmospheric dispersion modeling" refers to the mathematical simulation of atmospheric dispersion. Description of contaminant transport in the atmosphere is used to describe the combination of diffusion and advection that occurs within the air is analogous to the earth surface. The concentration of a contaminant released into the air may therefore be described by advection - diffusion equation by Stockie JM [14]. The advection - diffusion equation has been widely applied. In the operational atmospheric dispersion model to predict the meaning. Concentration of contaminants in the planetary boundary layer (PBL) which is obtaining the dispersion from a continuous point source by Tiziano T et al. [15]. For nearly thirty years, it has been known that vertical concentration profiles from field and laboratory experiments of near-surface points. Source releases exhibit a non-Gaussian distribution [16-18]. Describes the meteorological phenomena and mechanisms involved in the dispersion of atmospheric liquid waste, discusses ways in which concentration and precipitation may be calculated in the area and identifies the data required for the models. The diffusion has resolved in three dimensions using the separation of the variables technique to assess the concentration of pollutants for each emission rate, taking into account the eddy diffusivities of pollutants and wind velocity in a neutral state in [9]. [10] has obtained from the natural compound concentration of pollutants after solving the temporary diffusion equation using the separation method in view of the eddy diffusivities measured at night or at any time in the high-solubility layer in a stable state. The diffusion equation has solved in two-dimensional by the semi-infinite field by [2]. The analytical or numerical solutions, together with a preliminary case and two threshold conditions, help us to understand the behavior of the distribution of the concentration of contaminants through an open 
medium such as air, rivers, lakes and porous media such as the aquifer, in which remedial actions are designed to reduce damage elimination. While obtaining analytical solutions to dispersion problems under optimal conditions, the basic approach was to reduce the propagation equation by removing advection terms [1]. The Laplace conversion technique was applied to the Advection-Diffusion Equations (ADE) in two dimensions to obtain crosswind integrated normalized concentration, consider wind speed and the vertical eddy diffusivity are constant. Data set used from atmospheric diffusion experiments conducted in the northern part of Copenhagen, Denmark was observed for hexafluoride traceability $\left(\mathrm{SF}_{6}\right)$ Essa. [19]. It has used to solve the stable advection and propagation equation $2 \mathrm{D}$ continuous point source with vertical vortex propagation as force of law of vertical height and wind distance, using wind speed as a power law Essa. [21].

In the current work, the (ADE) is solved in two dimensions $(\mathrm{x}, \mathrm{z})$ to obtain the crosswind integrated concentration using the Laplace conversion technique with constant wind speed and vertical eddy diffusivity is variable with vertical distance. For trace hexafluoride $\left(\mathrm{SF}_{6}\right)$, comparisons were made between calculated values and observed crosswind integrated normalized concentrations in the northern part of Copenhagen, Denmark, as well as Inshas, Cairo, Egypt, under unstable conditions.

\section{Analytical Solution}

The transfer of the steady state to release non-reactive pollutants from a point source is described by following the partial differential equation [4].

$$
\mathrm{u} \frac{\partial \mathrm{C}}{\partial \mathrm{x}}=\frac{\partial}{\partial \mathrm{y}}\left(\mathrm{K}_{\mathrm{y}} \frac{\partial \mathrm{C}}{\partial \mathrm{y}}\right)+\frac{\partial}{\partial \mathrm{z}}\left(\mathrm{K}_{\mathrm{z}} \frac{\partial \mathrm{C}}{\partial \mathrm{z}}\right)
$$

$\mathrm{C}$ denotes the pollutant concentration, $\mathrm{K}_{\mathrm{z}}$ is the turbulent eddy diffusivity coefficient assumed to be a function of the variable $\mathrm{z}, \mathrm{u}$ is the mean wind oriented in the $\mathrm{x}$ direction which is constant.

Diffusion in the $\mathrm{x}$-direction, $\frac{\partial}{\partial \mathrm{x}}\left(\mathrm{K}_{\mathrm{x}} \frac{\partial \mathrm{C}}{\partial \mathrm{x}}\right)$ has been very small relative Advection $\left(\mathrm{u} \frac{\partial \mathrm{C}}{\partial \mathrm{x}}\right)$ therefore the term

$\frac{\partial}{\partial \mathrm{x}}\left(\mathrm{K}_{\mathrm{x}} \frac{\partial \mathrm{C}}{\partial \mathrm{x}}\right)$ has been neglected in comparison with Advection

The crosswind-integrated concentration $\left(\mathrm{C}_{\mathrm{y}}\right)$ is obtained by integrating equation (1) with respect to $y$ from $-\infty$ to $+\infty$ as follows:

$$
\mathrm{u} \frac{\partial \overline{\mathrm{C}}_{\mathrm{y}}}{\partial \mathrm{x}}=\frac{\partial}{\partial \mathrm{z}}\left(\mathrm{K}_{\mathrm{z}} \frac{\partial \overline{\mathrm{C}}_{\mathrm{y}}}{\partial \mathrm{z}}\right)
$$

Equation (2) becomes:

$$
\frac{\partial \overline{\mathrm{C}}_{\mathrm{y}}}{\partial \mathrm{x}}=\frac{\mathrm{K}_{\mathrm{z}}}{\mathrm{u}} \frac{\partial^{2} \overline{\mathrm{C}}_{\mathrm{y}}}{\partial \mathrm{Z}^{2}}+\frac{\mathrm{K}_{\mathrm{z}}{ }^{\prime}}{\mathrm{u}} \frac{\partial \overline{\mathrm{C}}_{\mathrm{y}}}{\partial \mathrm{Z}}
$$

Where $\mathrm{K}_{\mathrm{z}}{ }^{\prime}$ is differential Vertical dispersion coefficient with respect to $\mathrm{z}$.

Equation (3) was subject to following the boundary conditions:

1-The pollutant is released from an elevated source of strength $\mathrm{Q}$ located at $(0, \mathrm{~h})$ i.e.:

$$
\mathrm{u} \overline{\mathrm{C}}_{\mathrm{y}}(\mathrm{x}, \mathrm{z})=\mathrm{Q} \delta(\mathrm{z}-\mathrm{hs}) \text { at } \mathrm{x}=0
$$

Where " $\mathrm{h}_{\mathrm{s}}$ " is a stack height and $\delta()$ is the Dirac delta function.

2-The concentration of the pollutant tends to zero at large distance of the source i.e.:

$$
\overline{\mathrm{C}}_{\mathrm{y}}(\mathrm{x} . \mathrm{z})=0 \text { at } \mathrm{x} \rightarrow \infty, \mathrm{z} \rightarrow \infty
$$

3- The flux at the ground and the height of the planetary boundary layer can be given by:

$$
\mathrm{K}_{\mathrm{z}} \frac{\partial \overline{\mathrm{C}}_{\mathrm{y}}}{\partial \mathrm{z}}=0 \text { at } \mathrm{z}=0, \mathrm{~h}
$$

Where $h$ is the height of the planetary boundary layer (PBL),

Considering the vertical eddy diffusivity ' $\mathrm{K}_{\mathrm{Z}}$ " takes the form as [4].

$$
\mathrm{K}_{\mathrm{z}}=\frac{0.4 \mathrm{u}_{*} \mathrm{z}}{\left[1-\left(\frac{15 \mathrm{z}}{\mathrm{L}}\right)\right]^{1 / 4}}
$$

Where, $\mathrm{u} *$ is a friction velocity and $\mathrm{L}$ is the Monin -Obukhov length.

Substituting from equation (7) in Equation (3), one gets as follows:

$$
\frac{\partial \overline{\mathrm{C}}_{\mathrm{y}}}{\partial \mathrm{x}}=\frac{0.4 \mathrm{u}_{*} \mathrm{z}}{\mathrm{u}\left[1-\left(\frac{15 \mathrm{z}}{\mathrm{L}}\right)\right]^{1 / 4}} \frac{\partial^{2} \overline{\mathrm{C}}_{\mathrm{y}}}{\partial \mathrm{z}^{2}}+\frac{0.1 \mathrm{u}_{*}(45 \mathrm{z}-4 \mathrm{~L})}{\mathrm{u}(15 \mathrm{z}-\mathrm{L}) \sqrt[4]{1-\frac{15 \mathrm{z}}{\mathrm{L}}}} \frac{\partial \overline{\mathrm{C}}_{\mathrm{y}}}{\partial \mathrm{z}}
$$

Applying the Laplace transform on equation (8) to respect $\mathrm{x}$. one obtains:

$$
\begin{gathered}
\check{\mathrm{C}}(\mathrm{s}, \mathrm{z})=\mathrm{L}_{\mathrm{p}}\{\mathrm{x}(\mathrm{s}) ; \mathrm{x} \rightarrow \mathrm{s}\} \\
\mathrm{s} \widetilde{\overline{\mathrm{C}}_{\mathrm{y}}}(\mathrm{s}, \mathrm{z})-\mathrm{C}(0, \mathrm{z})= \\
\frac{0.4 \mathrm{u}_{*} \mathrm{z}}{\mathrm{U}\left[1-\left(\frac{15 \mathrm{z}}{\mathrm{L}}\right)\right]^{1 / 4}} \frac{\partial^{2} \widetilde{\overline{\mathrm{C}}_{\mathrm{y}}}(\mathrm{s}, \mathrm{z})}{\partial \mathrm{z}^{2}}+\frac{0.1 \mathrm{u}_{*}(45 \mathrm{z}-4 \mathrm{~L})}{\mathrm{u}(15 \mathrm{z}-\mathrm{L}) \sqrt[4]{1-\frac{15 \mathrm{z}}{\mathrm{L}}}} \frac{\partial \overline{\mathrm{C}}_{\mathrm{y}}(\mathrm{s}, \mathrm{z})}{\partial \mathrm{z}}
\end{gathered}
$$

Where " $\mathrm{s}$ " is the Laplace transform from $\mathrm{x}$ to $\mathrm{s}$

Equation (9) becomes:

$$
\begin{gathered}
\frac{0.4 \mathrm{u}_{*} \mathrm{z}}{\mathrm{u}\left[1-\left(\frac{15 \mathrm{z}}{\mathrm{L}}\right)\right]^{1 / 4}} \frac{\partial^{2} \widetilde{\overline{\mathrm{C}}_{\mathrm{y}}}(\mathrm{s}, \mathrm{z})}{\partial \mathrm{z}^{2}}+\frac{0.1 \mathrm{u}_{*}(45 \mathrm{z}-4 \mathrm{~L})}{\mathrm{u}(15 \mathrm{z}-\mathrm{L}) \sqrt[4]{1-\frac{15 \mathrm{z}}{\mathrm{L}}}} \frac{\partial \widetilde{\overline{\mathrm{C}}_{\mathrm{y}}}(\mathrm{s}, \mathrm{z})}{\partial \mathrm{z}}-\mathrm{s} \widetilde{\overline{\mathrm{C}}_{\mathrm{y}}}(\mathrm{s}, \mathrm{z})= \\
-\mathrm{C}(0, \mathrm{z})
\end{gathered}
$$

Applying the Laplace transforms on equation (6) of boundary condition with respect to $\mathrm{x}$, this becomes:

$$
\frac{0.1 u_{*}(45 z-4 L)}{u(15 z-L) \sqrt[4]{1-\frac{15 z}{L}}} \frac{\partial \widetilde{\bar{C}}_{y}(s, z)}{\partial z}=0
$$

The equation (10) becomes:

$$
\begin{gathered}
\left(\frac{\partial}{\partial z}-\sqrt{\frac{s u\left[1-\left(\frac{15 z}{L}\right)\right]^{1 / 4}}{0.4 u_{*} z}}\right)\left(\frac{\partial}{\partial z}+\sqrt{\frac{s u\left[1-\left(\frac{15 z}{L}\right)\right]^{1 / 4}}{0.4 u_{*} z}}\right) \widetilde{C}_{y}(s, z)= \\
-\frac{C(0, z)\left[1-\left(\frac{15 z}{L}\right)\right]^{1 / 4}}{0.4 u_{*} \mathrm{z}}
\end{gathered}
$$


Where,

$$
\frac{\mathrm{R}(\mathrm{x})}{\left(\mathrm{D}-\mathrm{m}_{1}\right)\left(\mathrm{D}+\mathrm{m}_{2}\right)}=\mathrm{e}^{\mathrm{m}_{1} \mathrm{z}} \int \mathrm{e}^{-\mathrm{m}_{1} \mathrm{z}} \mathrm{e}^{\mathrm{m}_{2} \mathrm{z}} \mathrm{R}(\mathrm{z}) \mathrm{dz}[11] .
$$

Where $\mathrm{R}=\frac{\mathrm{C}(0, \mathrm{z})\left[1-\left(\frac{15 \mathrm{z}}{\mathrm{L}}\right)\right]^{1 / 4}}{0.4 \mathrm{u}_{* \mathrm{z}} \mathrm{z}}, \mathrm{D}=\frac{\partial}{\partial \mathrm{z}}$,

$$
\mathrm{m} 1=-\sqrt{\frac{\mathrm{su}\left[1-\left(\frac{15 \mathrm{z}}{\mathrm{L}}\right)\right]^{1 / 4}}{0.4 \mathrm{u}_{*} \mathrm{z}}} \text { and } \mathrm{m} 2=+\sqrt{\frac{\mathrm{su}\left[1-\left(\frac{15 \mathrm{z}}{\mathrm{L}}\right)\right]^{1 / 4}}{0.4 \mathrm{u}_{*} \mathrm{z}}}
$$

Substituting from boundary condition equation (4) in equation (11) one gets:

$$
\frac{\partial^{2} \widetilde{\mathrm{C}_{\mathrm{y}}}(\mathrm{s}, \mathrm{z})}{\partial \mathrm{Z}^{2}}-\frac{\mathrm{su}\left[1-\left(\frac{15 \mathrm{z}}{\mathrm{L}}\right)\right]^{1 / 4}}{0.4 \mathrm{u}_{*} \mathrm{z}} \overline{\mathrm{C}}_{\mathrm{y}}(\mathrm{s}, \mathrm{z})=-\frac{\mathrm{Q} \delta\left(\mathrm{z}-\mathrm{h}_{\mathrm{s}}\right)\left[1-\left(\frac{15 \mathrm{z}}{\mathrm{L}}\right)\right]}{0.4 \mathrm{u}_{*} \mathrm{z}}
$$

The solution of equation (12) becomes:

$$
\widetilde{\bar{C}_{y}}(s, z)=\frac{Q\left[1-\left(\frac{15 h_{s}}{L}\right)\right]^{1 / 4}}{0.4 u_{*} h_{s}} e^{-\sqrt{s} \sqrt{\frac{U\left[1-\left(\frac{15 z}{L}\right)\right]^{1 / 4} z}{0.4 u_{*}}}}
$$

Apply the Laplace inverse transform on equation (13) to respect $\mathrm{x}$, one obtains that:

$$
\frac{\overline{\mathrm{C}}_{\mathrm{y}}(\mathrm{x}, \mathrm{z})}{\mathrm{Q}}=\frac{\left[1-\left(\frac{15 \mathrm{~h}_{\mathrm{s}}}{\mathrm{L}}\right)\right]^{1 / 4}}{0.4 \mathrm{u}_{*} \mathrm{~h}_{\mathrm{s}}}\left(\sqrt{\frac{\mathrm{u}\left[1-\left(\frac{15 \mathrm{z}}{\mathrm{L}}\right)\right]^{1 / 4} \mathrm{z}}{0.4 \mathrm{u}_{*} \pi \mathrm{x}}} \mathrm{e}^{-\frac{\mathrm{u}\left[1-\left(\frac{15 \mathrm{z}}{\mathrm{L}}\right)\right]^{1 / 4} \mathrm{z}}{0.16 \mathrm{xu}_{*}}}\right)
$$

and

$$
\mathrm{C}(\mathrm{x}, \mathrm{y}, \mathrm{z})=\overline{\mathrm{C}}_{\mathrm{y}}(\mathrm{x}, \mathrm{z})\left(\frac{\mathrm{e}^{-\frac{\mathrm{y}^{2}}{2 \sigma_{\mathrm{y}}{ }^{2}}}}{\sqrt{2 \pi} \sigma_{\mathrm{y}}}\right)
$$

The equation (15) becomes:

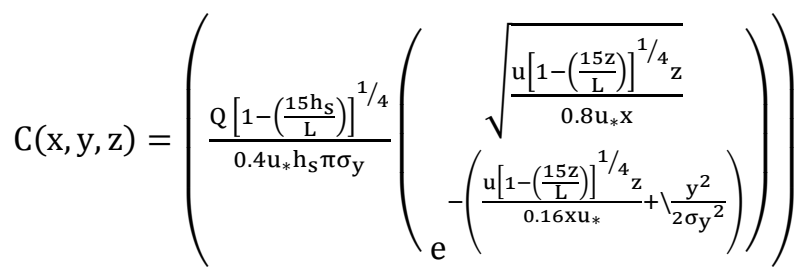

\section{Results and Discussion}

Copenhagen dispersion experiments. In these experiments, the pollutant was released at an altitude of 115 meters above the ground and the concentrations of pollutants were measured at ground level at 3 arcs located at a distance of 2000 to 6000 meters from the continuous elevation point of the source. Moreover, the Copenhagen data set provides data on wind and turbulence at 10 and 115 meters. The motivation for choosing the Copenhagen experiment was the fact that the area in which the experiment was conducted was located near the coast of resound. [6, 7].

Comparison between Observed and calculated, Ref. [20] crosswind-integrated concentration $\overline{\mathrm{C}}_{\mathrm{y}}(\mathrm{x}, \mathrm{z})$ at different stability, height, Wind Speed and, Distance in Copenhagen, Denmark (See in Table 1). Meteorological data (downwind distance, Wind speed, stability classes and effective heights, emission rate, friction velocity, Monin -Obukhov length) and a comparison between our results and the results obtained from Inshas, Cairo, Egypt (See in Table 1).

Explanation of the statistical evaluation of model results (See in Table 3). Scatter diagram between observed and calculated $\overline{\mathrm{C}}_{\mathrm{y}}(\mathrm{x}, \mathrm{z})$ for the (A) Copenhagen experiments (B) Inshas, Cairo,

\begin{tabular}{|c|c|c|c|c|c|c|c|}
\hline \multirow{2}{*}{ Run no. } & \multirow{2}{*}{ Stability } & \multirow{2}{*}{ H (m) } & \multirow{2}{*}{$\mathbf{u} *(\mathbf{m} / \mathbf{s})$} & \multirow{2}{*}{ X (m) } & \multicolumn{3}{|c|}{$\frac{\bar{C}_{y}}{Q}\left(10^{-4} \mathrm{~s} / \mathrm{m}^{2}\right)$} \\
\hline & & & & & Observed & Refs.[20] & Predicted \\
\hline 1 & $\mathrm{~A}$ & 1980 & 3.03 & 1900 & 6.48 & 7.705 & 5.49 \\
\hline 1 & A & 1980 & 3.03 & 3700 & 2.31 & 3.488 & 2.78 \\
\hline 2 & B & 1920 & 7.99 & 2100 & 5.38 & 4.620 & 4.75 \\
\hline 2 & $\mathrm{C}$ & 1920 & 7.99 & 4200 & 2.95 & 2.307 & 6.76 \\
\hline 3 & A & 1120 & 3.46 & 1900 & 8.2 & 8.411 & 4.43 \\
\hline 3 & $\mathrm{D}$ & 1120 & 3.46 & 3700 & 6.22 & 3.221 & 1.00 \\
\hline 3 & $\mathrm{E}$ & 1120 & 3.46 & 5400 & 4.3 & 1.614 & 1.59 \\
\hline 5 & $\mathrm{C}$ & 820 & 4.08 & 2100 & 6.72 & 6.580 & 7.62 \\
\hline 5 & A & 820 & 5.05 & 4200 & 5.84 & 2.044 & 1.25 \\
\hline 5 & $\mathrm{D}$ & 820 & 5.05 & 6100 & 4.97 & 1.004 & 1.87 \\
\hline 6 & B & 1300 & 5.05 & 2000 & 3.96 & 3.752 & 5.01 \\
\hline 6 & A & 1300 & 11.73 & 4200 & 2.22 & 1.704 & 3.01 \\
\hline 6 & A & 1300 & 11.73 & 5900 & 1.83 & 1.005 & 1.01 \\
\hline 7 & $\mathrm{~A}$ & 1850 & 11.73 & 2000 & 6.7 & 5.917 & 5.49 \\
\hline 7 & A & 1850 & 5.91 & 4100 & 3.25 & 2.893 & 2.78 \\
\hline 7 & B & 1850 & 5.91 & 5300 & 2.23 & 2.124 & 4.75 \\
\hline 8 & $\mathrm{C}$ & 810 & 5.91 & 1900 & 4.16 & 7.125 & 6.76 \\
\hline 8 & A & 810 & 7.73 & 3600 & 2.02 & 3.124 & 4.43 \\
\hline
\end{tabular}
Egypt. Dashed lines indicate factor of 2 (See in Figure1). Comparison between crosswind- normalized integrated concentration and downwind distance for the (C) Copenhagen experiments (D) Inshas, Cairo, Egypt (See in Figure 2).

Table 1. Meteorological data (downwind distance, stability classes and effective heights, emission rate, friction velocity, Monin -Obukhov length) Observed and calculated Crosswind-integrated concentration $\bar{C}_{y}(x, z)$ per emission rate at different stability, height, Wind Speed and, Distance in Copenhagen, Denmark. 


\begin{tabular}{|c|c|c|c|c|c|c|c|}
\hline \multirow{2}{*}{ Run no. } & \multirow{2}{*}{ Stability } & \multirow{2}{*}{$\mathbf{H}(\mathbf{m})$} & \multirow{2}{*}{$\mathbf{u}_{*}(\mathbf{m} / \mathbf{s})$} & \multirow{2}{*}{$X(m)$} & \multicolumn{3}{|c|}{$\frac{\overline{\bar{C}_{y}}}{\mathrm{Q}}\left(10^{-4} \mathrm{~s} / \mathrm{m}^{2}\right)$} \\
\hline & & & & & Observed & Refs.[20] & Predicted \\
\hline 8 & $\mathrm{D}$ & 810 & 7.73 & 5300 & 1.52 & 1.519 & 1.00 \\
\hline 9 & E & 2090 & 7.73 & 2100 & 4.58 & 4.902 & 1.59 \\
\hline 9 & $\mathrm{C}$ & 2090 & 8.31 & 4200 & 3.11 & 2.485 & 7.62 \\
\hline 9 & $\mathrm{~A}$ & 2090 & 8.31 & 6000 & 2.59 & 1.682 & 1.25 \\
\hline
\end{tabular}

A

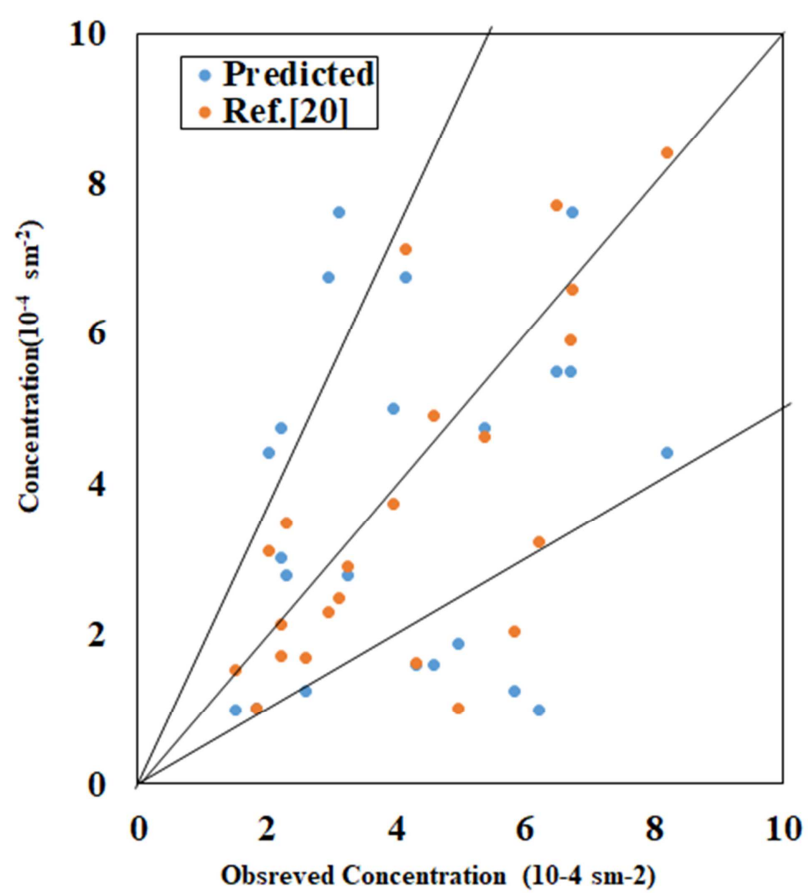

B

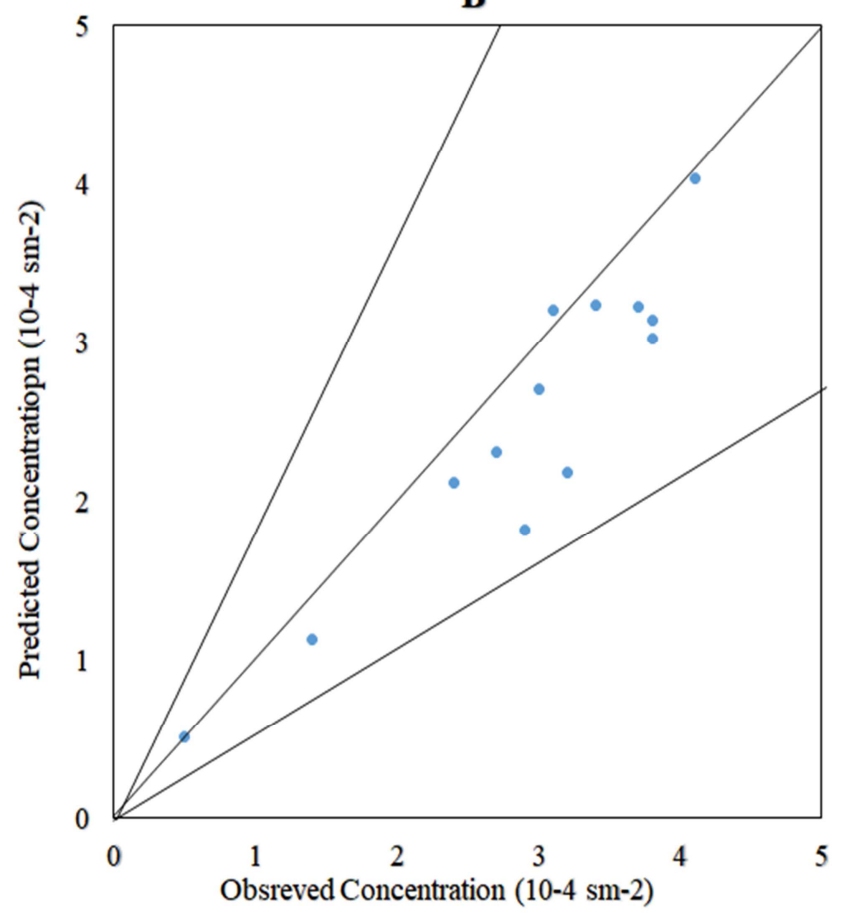

Figure 1. Scatter diagram between observed and calculated $\bar{C}_{y}(A, z)$ for the (A) Copenhagen experiments (B) Inshas, Cairo, Egypt. Dashed lines indicate factor of 2 .

A

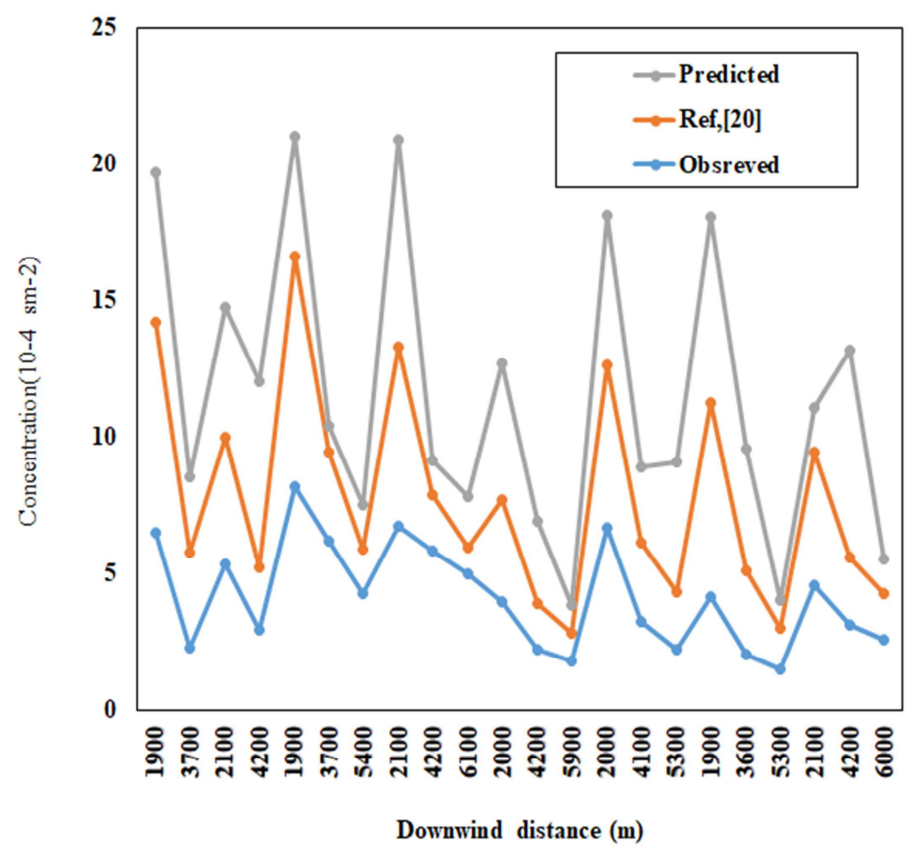

B

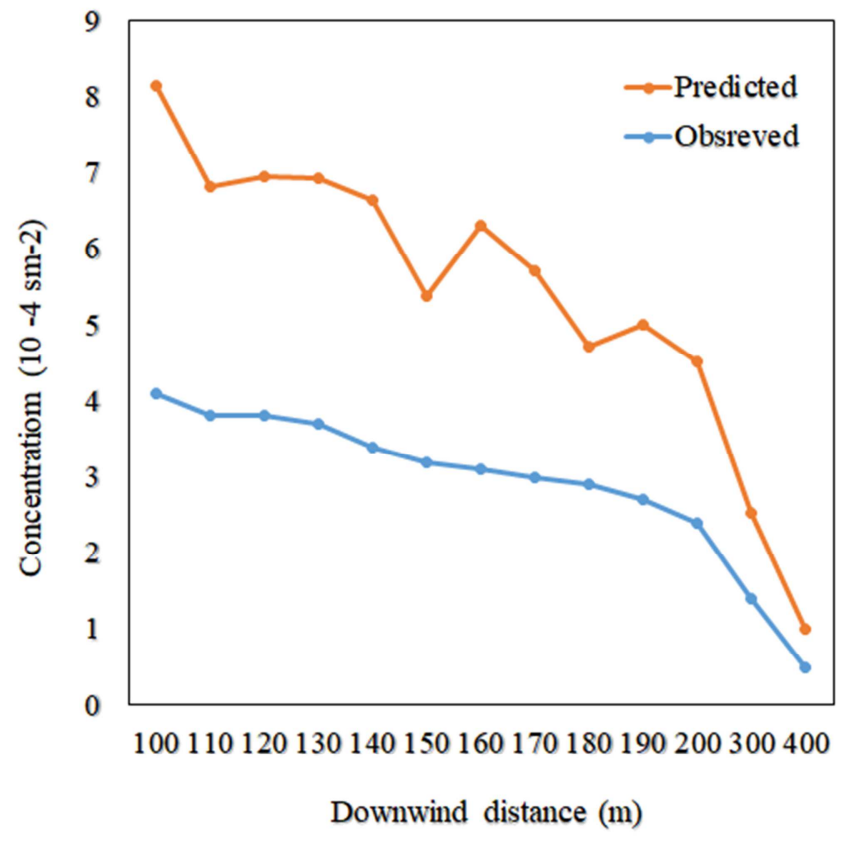

Figure 2. Comparison between crosswind-normalized integrated concentration and downwind distance for the (A) Copenhagen experiments (B) Inshas, Cairo, Egypt. 
Table 2. Shows that the meteorological data (downwind distance ' $x$ ', wind speed ' $u$ ') stability classes and different effective heights for two height stacks (H)(43m and $27 \mathrm{~m}$ ) respectively for different emission rate at Inshas, Egypt.

\begin{tabular}{llllllll}
\hline Exp. & $\mathbf{Q}$ & $\mathbf{x}(\mathbf{m})$ & $\mathbf{u}_{*}(\mathbf{m} / \mathbf{s})$ & $\mathbf{L}(\mathbf{m})$ & $\mathbf{H}(\mathbf{m})$ & Observed & Predicted \\
\hline 1 & 28114286 & 100 & 0.4 & -198 & 49 & 4.1 & 4.033 \\
2 & 28700000 & 110 & 0.26 & -198 & 48 & 3.8 & 3.019 \\
3 & 1171429 & 120 & 0.27 & -192 & 45 & 3.8 & 3.143 \\
4 & 12885714 & 130 & 0.26 & -192 & 46 & 3.7 & 3.223 \\
5 & 13471429 & 140 & 0.2 & -112 & 45 & 3.4 & 2.184 \\
6 & 140557143 & 150 & 0.26 & -112 & 45 & 3.2 & 3.204 \\
7 & 27528571 & 160 & 0.3 & -112 & 47 & 3.1 & 2.708 \\
8 & 28524286 & 170 & 0.4 & -82 & 46 & 3.0 & 2.818 \\
9 & 28260714 & 180 & 0.26 & -82 & 47 & 2.9 & 2.310 \\
10 & 2928571.4 & 190 & 0.27 & -82 & 28 & 2.7 & 1.131 \\
11 & 4100000 & 200 & 0.26 & -130 & 28.3 & 2.4 & 0.506 \\
12 & 1171428.6 & 300 & 0.2 & -130 & 30.8 & 1.4 & \\
13 & 2342857.1 & 400 & 0.26 & -130 & 30.6 & 0.5 & \\
\hline
\end{tabular}

\section{Statistical Method}

The statistical indicators in Table 3 are the following [5].

$$
\begin{gathered}
\text { Fraction Bias }(\mathrm{FB})=\frac{\left(\overline{\mathrm{C}_{\mathrm{o}}}-\overline{\mathrm{C}_{\mathrm{p}}}\right)}{\left[0.5\left(\overline{\mathrm{C}_{\mathrm{o}}}+\overline{\mathrm{C}_{\mathrm{p}}}\right)\right]} \\
\text { Normalized Mean Square Error (NMSE) }=\frac{\overline{\left(\mathrm{C}_{\mathrm{p}}-\mathrm{C}_{\mathrm{o}}\right)^{2}}}{\overline{\left(\mathrm{C}_{\mathrm{p}} \mathrm{C}_{\mathrm{o}}\right)}} \\
\text { Correlation Coefficient (COR) }=\frac{1}{\mathrm{~N}_{\mathrm{m}}} \sum_{\mathrm{i}=1}^{\mathrm{N}_{\mathrm{m}}}\left(\mathrm{C}_{\mathrm{pi}}-\overline{\mathrm{C}_{\mathrm{p}}}\right) \times \frac{\left(\mathrm{C}_{\mathrm{oi}}-\overline{\mathrm{C}_{\mathrm{o}}}\right)}{\left(\sigma_{\mathrm{p}} \sigma_{\mathrm{o}}\right.} \\
\text { Factor of Two }(\mathrm{FAC} 2)=0.5 \leq \frac{\mathrm{C}_{\mathrm{p}}}{\mathrm{C}_{\mathrm{o}}} \leq 2.0
\end{gathered}
$$

Where $\mathrm{C}$ is the quantity analyzed (concentration) and the values "o" and "p" represent the observed values and present values, respectively. Extra lines in statistical indicators indicate averages. The FB statistic indicates whether the expected quantity reduces the amount observed or overestimates it. The NMSE statistical indicator represents the squared error of the expected quantity with respect to the observed quantity. The statistical indicator FA2 provides a small portion of the data for $0.5 \leq \mathrm{Cp} / \mathrm{C} 0 \leq 2.0$ as the nearest zero are NMSE, FB and FS and the closest one is R and FA2, best results.

According to Tables 1 and 2 and Figures 1 and 2, the results showed a satisfactory agreement between measurements and simulations. The values of NMSE and FB are relatively close to zero, and COR, FA2 are relatively close to one.

Table 3. Statistical evaluation of models results.

\begin{tabular}{lllll}
\hline Models & NMSE & FB & COR & FAC2 \\
\hline Inshas, Cairo, Egypt & 0.21 & 0.13 & 0.97 & 0.96 \\
Predicted (Copenhagen, Denmark) & 0.41 & 0.14 & 0.98 & 0.97 \\
Ref. [20](Copenhagen, Denmark) & 0.18 & 0.1 & 0.64 & 0.95 \\
\hline
\end{tabular}

\section{Conclusion}

The diffusion equation was mathematically derived and we compared the results obtained from the conclusion with other results, namely the Copenhagen experiment and Egypt Inshas. We performed a statistical method to evaluate them, and found that the results are within a factor of two, NMSE and FB values are relatively close to zero, and COR, FA2 are relatively close to one.

\section{Acknowledgements}

All thanks go back to God Almighty who threatened me and helped me accomplish this work I would like to thank Prof. Dr. Khaled Sadek Mohamed Essa for his help.

\section{References}

[1] Amruta Daga, V. H. (2013). Analytical solution of advection diffusion equation in homogeneous medium. International journal of science, spirituality, business and technology (ijssbt), vol. 2 , no. $1,2277-7261$.

[2] Chatterjee, A. and Singh, M. K. (2018) Two-dimensional advection -dispersion equation with depth-dependent variable source concentration. Pollution, 4 (1): 1-8.

[3] Demuth, C., (1978). "A contribution to the analytical steady solution of the diffusion equation". Atom. Environ. 12, 1255. 
[4] Hanna Steven R. Gary A. Briggs and Rayford P. hosker. Jr. (1982) Handbook on atmospheric diffusion. Technical Information Center, U. S. Department of Energy.

[5] Hanna, S. R., (1989) Confidence limit for air quality models as estimated by bootstrap and Jackknife resembling methods. Atom. Environ. 23, 1385-1395.

[6] Gryning. S. E., and Lyck., (1984) Atmospheric dispersion from elevated sources in an urban area: Comparison between tracer experiments and model calculations. J. Climate Appl. Meteor., 23, pp. 651-660.

[7] Gryning, S. E., Holtslag, A. A. M., Irwin, J. S., Sivertsen, B. (1987) Applied dispersion modeling based on meteorological scaling parameters. Atmos. Environ. 21 (1), 79-89.

[8] John M. (2011) The mathematical of atmospheric dispersion modeling. Society for Industrial Applied Mathematics. 53. 349-372.

[9] Khaled S. M Essa and S. E. M. Elsaid (2015) Solving the advection diffusion equation in three dimensions in neutral case. Pyrex Journal of Ecology and the Natural Environment Vol 1 (1) pp. 001-006.

[10] Khaled Sadek Mohamed Essa, Sawsan Ibrahim Mohamed El Saied, Ayman Marrouf (2018) Analytical Solution of Time Dependent Diffusion Equation in Stable Case. American Journal of Environmental Science and Engineering; 2 (2): 32-36.

[11] Murray R. Spiegel (1992) Advanced mathematics for engineers and scientists. McGill Publishing House.

[12] Demuth C (1978) A contribution to the analytical steady solution of the diffusion equation. Atoms Environ 12: 1255-1978 2.

[13] Essa KSM (2014) Studying the effect of vertical eddy diffusivity on the solution of diffusion equation. Physical science international Journal 4: 355-365.

[14] Stockie JM (2011) The mathematics of atmospheric dispersion modeling. Society for industrial and applied mathematics 5: 349-372.

[15] Tiziano T, Moreira DM, Vilhena MT, Costa CP (2010) Comparison between non- Gaussian puff model and a model based on a time-dependent solution of advection equation. Journal of Environment protection 1: 172-178.

[16] Elliot WP (1961) The vertical diffusion of gas from continuous source. Int $\mathrm{j}$ air water pollut 4: 33-46.

[17] Malhorta RC, Cermak JE (1964) Mass diffusion in neutral and unstably stratified boundary-layer flouss. J Heat mass trans 7: 169-186.

[18] Marrouf AA, Mohamed AS, Ismail G, Essa KSM (2013) An analytical solution of two dimensional atmospheric diffusion equation in a finite boundary layer. International Journal of Advanced Research Volume 1: 356-365.

[19] Khaled Sadek Mohamed Essa, Sawsan Ibrahim Mohamed El Saied (2019) Mathematical Solution of Two Dimensional Advection-Diffusion Equations. Journal of Chemical, Environmental and Biological Engineering Volume 3, Issue 1, Pages: 8-12.

[20] Essa KSM, Mina AN, Hamdy HS and Khalifa AA (2016) Theoretical Solution of the Diffusion Equation in Unstable Case. Int J Account Res, 4: 1.

[21] Khaled S. M. Essa, Ahmed S. Shalaby, Mahmoud A. E. Ibrahim, And Ahmed M. Mosallem1 2020) Analytical Solutions of the Advection-Diffusion Equation with Variable Vertical Eddy Diffusivity and Wind Speed Using Hankel Transform. Pure Appl. Geophys. 177, 4545-4557. 